\title{
The radio spectral index of the Vela supernova remnant
}

\author{
H. Alvarez ${ }^{1}$, J. Aparici ${ }^{1}$, J. May $^{1}$, and P. Reich ${ }^{2}$ \\ 1 Departamento de Astronomía, Universidad de Chile, Casilla 36-D, Santiago, Chile \\ 2 Max-Planck-Institut für Radioastronomie, Auf dem Hügel 69, 53121 Bonn, Germany
}

A\&A, 372, 636-643 (2001), DOI: 10.1051/0004-10361:20010509

Figure 6 on page 642 was of low quality. So we print it again.

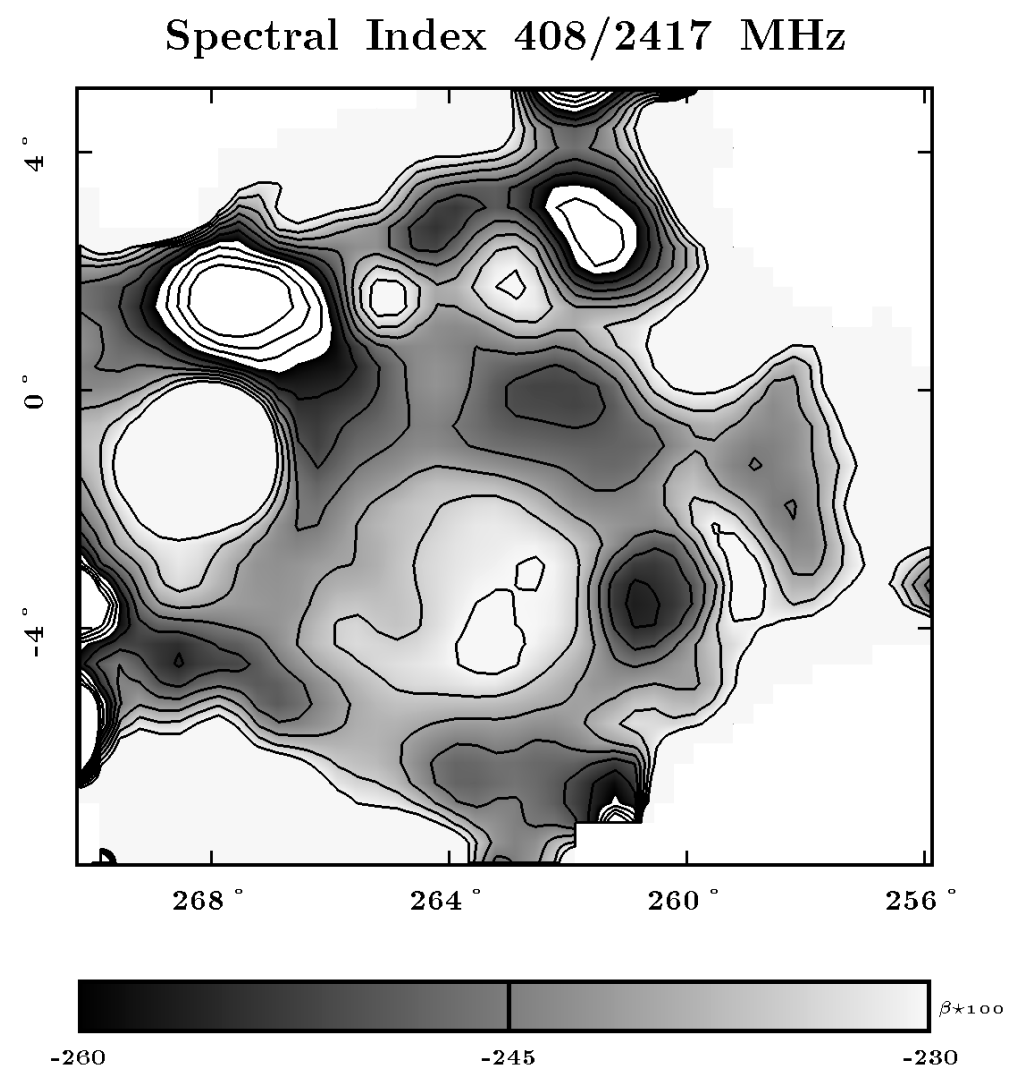

Fig. 1. Brightness temperature spectral index $(408 / 2417 \mathrm{MHz})$ over the Vela-Puppis area. The map shows a circular distribution around Vela $\mathrm{X}\left(264^{\circ} .0,-3^{\circ} 3\right)$. This structure can de produced by a source with index $\beta \sim-2.3$, at the centre, superimposed on a larger circular area with a steeper $(\beta \sim-2.6)$ and uniform spectrum. The source at $\left(268^{\circ},-1^{\circ}\right)$ is the HII region RCW 38 . The gray scale starts at $\beta=-2.3$ (white) and runs to $\beta=-2.6$ (black). The contours start at -2.3 in steps of -0.05 .

Send offprint requests to: H. Alvarez, alvarez@das.uchile.cl 